\title{
Dispersive radiation induced by shock waves in passive resonators
}

\author{
Stefania Malaguti ${ }^{1}$, Matteo Conforti ${ }^{2}$, Stefano Trillo ${ }^{1}$ \\ ${ }^{1}$ Department of Engineering, University of Ferrara, Via Saragat 1, 44122 Ferrara, Italy \\ ${ }^{2}$ PhLAM/IRCICA, CNRS-Université Lille 1, UMR 8523/USR 3380, F-59655 Villeneuve d'Ascq, France
}

Corresponding author: stefania.malaguti@unife.it

Compiled June 3, 2014

We show that passive Kerr resonators pumped close to zero dispersion wavelengths on the normal dispersion side develop the resonant generation of linear waves driven by cavity (mixed dispersive-dissipative) shock waves. The resonance mechanism can be successfully described in the framework of the generalized Lugiato-Lefever equation with higher-order dispersive terms. Substantial differences with radiation from cavity solitons and purely dispersive shock waves dispersion are highlighted. (C) 2014 Optical Society of America

OCIS codes: (190.1450) Bistability; (060.4370) Nonlinear optics, fibers; (190.5530) Pulse propagation and temporal

solitons; (230.5750) Resonators

Optical temporal solitons excited close to a zerodispersion wavelength (ZDW) are well known to resonantly radiate dispersive waves across the ZDW in the spectral region characterized by a normal groupvelocity dispersion (GVD), owing to higher-order dispersion (HOD) [1,2]. In photonic crystal fibers such resonant radiation $(\mathrm{RR})$ can interact with the solitons, deeply affecting the blue-shifted region of the spectrum in supercontinuum generation [2]. Soliton-driven RR can also be deeply shifted in the UV spectral region in gas filled hollow-core fibers [3] or red-shifted in planar photonic crystal waveguides where the normal GVD regime corresponds to positive wavelength detunings [4]. More recently the studies of the RR have been extended to cavity solitons, i.e. solitons of passive resonator structures [5]. The resonance mechanism has been theoretically investigated for microresonators [6], and independently observed in a fiber ring experiment [7], while the related issue concerning the impact of HOD on frequency comb generation is a topic currently debated $[8,9]$. Such phenomena can be successfully accounted for in the framework of the so-called Lugiato-Lefever equation (LLE) [10], which provides an accurate mean-field description for the propagation in fiber rings $[11,12]$ as well as planar microresonators [13-16].

The aim of this Letter is to show that the emission of RR spectral peaks in passive resonators must be expected also when pumping in the normal GVD regime. Dispersive wave emission occurs, in this case, from the fronts associated with cavity dispersive shock waves (CDSW) traveling at definite velocity. DSWs are fast oscillating wavetrains that spontaneously develop over pulse tails that become steepened via the Kerr effect, owing to the regularizing action of a weak normal GVD [17-19]. As pointed out recently for the free (cavity-less) propagation along a fiber, higher-order dispersive corrections to GVD make DSWs to resonantly radiate. The dynamics of the radiation process is quite rich [20,21], encompassing a regime recently observed in a fiber experiment [22]. Here we extend the theory of shock-induced RR to passive resonators. In this case shock waves have, in general, mixed dissipative-dispersive nature, with the dispersive (oscillating) features prevailing in the low loss regime [23]. Here we show that, in the framework of the generalized LLE with HOD, these shock waves radiate. A good estimate of the radiated frequency can be given by means of perturbation theory $[2,6,26]$. We present numerical tests of this approach, also pointing out at the crucial role of the front velocity compared with the soliton case. Finally we emphasize that RR can act along with cavity modulation instability (MI) to determine non trivial spectral features. We specifically focus on the effect of third-order dispersion (TOD), where our results constitute novel manifestations of TOD-induced symmetry-breaking $[24,25]$.

We start from an averaged model of the cavity, namely a generalized LLE, expressed in dimensionless units as

$$
i \varepsilon \frac{\partial u}{\partial z}+d\left(i \varepsilon \partial_{t}\right) u+|u|^{2} u=[\delta-i \alpha] u+i \sqrt{P}
$$

where we adopt the normalisation introduced in Ref. [23]. In particular, the real world distance $Z$, time $T$, and intracavity field $E$ can be derived as $Z=z \sqrt{L L_{d}}$, $T=T_{0} t, E(T, Z)=u(t, z) / \sqrt{\gamma L}$, where $\gamma=k_{0} n_{2 I} / A_{\text {eff }}$ is the standard fiber nonlinear coefficient, $L$ and $L_{d}=$ $T_{0}^{2} / k^{\prime \prime}$ are the fiber (cavity) length and the dispersion length associated with time scale $T_{0}$ and GVD $k^{\prime \prime}=$ $d^{2} k / d \omega^{2}$, respectively. The cavity parameters are the detuning $\delta$ and the loss coefficient $\alpha=1-\rho \simeq \theta^{2} / 2$ due to output coupling with transmissivity $\theta\left(\rho^{2}=\right.$ $1-\theta^{2}$ ), while $P=\theta^{2} \gamma L P_{i n}$ is related to the dimensional input power $P_{i n}$. Furthermore $\varepsilon=\sqrt{L / L_{d}} \ll 1$ quantifies the smallness of GVD and HOD introduced through the operator $d\left(i \varepsilon \partial_{t}\right)=\sum_{n \geq 2} \beta_{n}\left(i \varepsilon \partial_{t}\right)^{n} / n !=$ $-\beta_{2} \varepsilon^{2} \partial_{t}^{2} / 2-i \beta_{3} \varepsilon^{3} \partial_{t}^{3} / 6+\ldots$, where the coefficients $\beta_{n}=$ $\partial_{\omega}^{n} k / \sqrt{(L)^{n-2}\left(\partial_{\omega}^{2} k\right)^{n}}\left[\right.$ note that $\left.\beta_{2}=\operatorname{sign}\left(\partial_{\omega}^{2} k\right)\right]$ are related to real-world HOD $\partial_{\omega}^{n} k$.

In the normal GVD regime $\left(\beta_{2}=1\right)$, wave-breaking induces the formation of moving fronts associated with $\mathrm{C}$ DSWs [23]. When C-DSWs are excited close to a ZDW, they are expected to radiate owing to resonances induced by HOD, in analogy to the conservative case [20,21]. The radiation frequency can be found by means of a perturbation approach $[2,6,26]$. Let us assume the main front of a C-DSW supported by leading order dispersion $\partial_{\omega}^{2} k$ 
to move with velocity $V=d z / d t$, i.e. $u=u_{s}(\tau)$, where $\tau=t-z / V$ is the retarded time in the shock frame (note that, having written Eq. (1) in the frame traveling at natural group velocity $V_{g}, 1 / V$ is proportional to the real-world group-velocity mismatch $1 / V_{\text {shock }}-1 / V_{g}$, i.e. the smaller $V$, the larger the difference between $V_{\text {shock }}$ and $V_{g}$ ). By substituting a perturbed intracavity field $u=u_{s}(\tau)+p(z, \tau)$ in Eq. (1), we obtain, after linearization $\left(|p| \ll\left|u_{s}\right|\right)$, the evolution equation for $p$

$$
i \varepsilon \frac{\partial p}{\partial z}+\hat{d}\left(i \varepsilon \partial_{\tau}\right) p+\left(2\left|u_{s}\right|^{2}-\delta+i \alpha\right) p+u_{s}^{2} p^{*}=F
$$

where $F=-\left[d\left(i \varepsilon \partial_{\tau}\right)-\beta_{2}\left(i \varepsilon \partial_{\tau}\right)^{2} / 2\right] u_{s}$ is a forcing term with zero wavenumber, and $\hat{d}\left(i \varepsilon \partial_{\tau}\right) \equiv$ $d\left(i \varepsilon \partial_{\tau}\right)-i \frac{\varepsilon}{V} \partial_{\tau}$. Setting $p(z, \tau)=A(z) \exp [i(k z-\omega \tau)]+$ $B^{*}(z) \exp [-i(k z-\omega \tau)]$, we find that the evolution of Stokes and antiStokes free wave amplitudes $a(z)=$ $[A(z) B(z)]^{T}$ is ruled by the system

$$
\begin{aligned}
& i \varepsilon \frac{d a}{d z}+C a=0, \\
& C=\left(\begin{array}{cc}
D(\varepsilon \omega)-\varepsilon k & u_{s}^{2} \\
-\left(u_{s}^{*}\right)^{2} & -D^{*}(-\varepsilon \omega)-\varepsilon k
\end{array}\right),
\end{aligned}
$$

where $D(\varepsilon \omega)=\tilde{d}(\varepsilon \omega)+2\left|u_{s}\right|^{2}-\delta+i \alpha$, and $\tilde{d}(\varepsilon \omega)$ is the Fourier transform of $\hat{d}\left(i \varepsilon \partial_{t}\right)$. The dispersion relation $k=k(\varepsilon \omega)$ of such waves is found by imposing $\operatorname{det}(C)=$ 0 , which yields two branches $k=k_{ \pm}(\varepsilon \omega)$. A resonance between such linear waves and the forcing front occurs at frequencies $\omega=\omega_{R R}$ such that $k_{ \pm}=k\left(\varepsilon \omega_{R R}\right)=0$. Due to the symmetry of the problem the RR frequencies arises in symmetric pairs $\pm\left|\omega_{R R}\right|$, which can be viewed as due to the four-wave mixing process. While this approach permits to treat HOD at any order, henceforth we focus on the effect of $\operatorname{TOD}\left(\beta_{3} \neq 0, \beta_{n}=0, n \geq 4\right)$, which is usually the dominant term. In this case, by posing $\left|u_{s}\right|^{2}=P_{u H}$ (which represents, the power of the higher state of the front, where RR is shed, as we show below), we explicitly obtain, in the limit of small losses $\alpha$,

$\varepsilon k_{ \pm}=\beta_{3} \frac{(\varepsilon \omega)^{3}}{6}-\frac{(\varepsilon \omega)}{V} \pm \sqrt{\left(\beta_{2} \frac{(\varepsilon \omega)^{2}}{2}+2 P_{u H}-\delta\right)^{2}-P_{u H}^{2}}$

A sufficiently accurate expression for the RR frequency can be obtained by expanding the square root in Eq. (5) at first order in $P_{u H}$. Then the main (stronger) peak of $\mathrm{RR}$ is found at the frequency $\omega=\omega_{R R}$ which solves the equation (equivalent to $k_{+}=0$ )

$$
\left[\beta_{3} \frac{(\varepsilon \omega)^{3}}{6}+\beta_{2} \frac{(\varepsilon \omega)^{2}}{2}-\frac{(\varepsilon \omega)}{V}-\delta\right]+2 P_{u H}=0 .
$$

Equation (6) is susceptible of a transparent physical interpretation, and could have been written directly on physical ground (see e.g. Ref. [21] for the conservative case). Indeed the term under square bracket represents the wavenumber of linear waves while the remaining term stands for the nonlinear correction induced through cross-phase modulation from the high-intensity plateau over which radiation is emitted. However, the present approach has the advantage to allow for the calculation
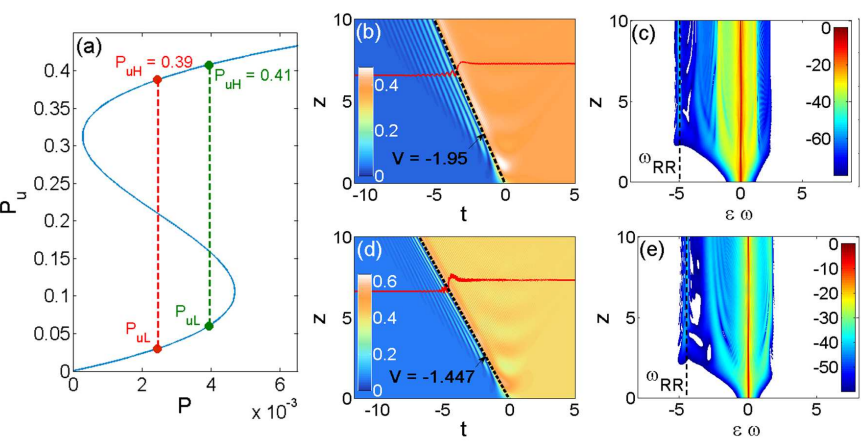

Fig. 1. (Color online) RR ruled by Eq. (1) starting from a step-like field connecting steady solutions on lower and upper branches of bistable response for two different values of $P$, as shown in (a). Temporal $(\mathrm{b}, \mathrm{d})$ and spectral (c,e) dynamics of $|u(t, z)|^{2}$. Upper row (b,c) and lower row $(\mathrm{d}, \mathrm{e})$ panels are relative to the jump with $P_{u H}=0.39$ and $P_{u H}=0.41$, respectively. Here $\varepsilon=0.1, \delta=\pi / 10, \alpha=0.03$, and $\beta_{3}=0.5$.

of the ratio between the amplitudes of the two RR components at opposite detunings $\omega=\omega_{R R}$. From the eigenvectors corresponding to eigenvalues $(5)$, we find

$$
\left|\frac{A}{B}\right| \simeq \frac{3\left|\beta_{2}\right|}{\left|\beta_{3}\right|} \frac{1}{P_{u}}\left|\frac{3 \beta_{2}^{2}}{\beta_{3}}-\frac{2}{V}\right|
$$

The validity of Eq. (6) can be conveniently tested by investigating the decay of an intracavity jump between two stationary solutions lying on different branches of the bistable response. To this end, we integrate numerically Eq. (1) by launching a nearly piecewise constant field $u(z=0, t)$ with values $u(t<0)=u_{L}$ (power $\left.P_{u}=P_{u L}\right)$ and $u(t>0)=u_{H}$ (power $\left.P_{u}=P_{u H}\right)$ lying on the low and high transmissive steady-states, respectively, and sustained by the same CW driving field with power $P$ [see Fig. 1(a)]. This condition is equivalent to assume that a steep gradient is formed directly at $z=0$. For small loss coefficients $\alpha$, dispersive effects dominate and the step-like intracavity field decays, owing to GVD, into a C-DSW featuring expanding oscillations that develop inside a shock fan, as shown in Fig. 1(b) and (d) for two choices of driving power $P$. The fan is delimited by a leading edge (where oscillations becomes vanishingly small, i.e. linear) and a trailing edge (deepest oscillation). In the presence of $\beta_{3} \neq 0$, the RR turns out to be emitted in the proximity of the trailing edge of the $\mathrm{C}$ DSW. Therefore its phase-matching condition is affected by the velocity $V$ of the latter, which is highlighted (see oblique dashed lines) in Fig. 1(b,d). By comparing Fig. 1 (b) and Fig. 1(d), it is clear that such velocity $V$ can change considerably, even for fixed values of the cavity parameters $\alpha$ and $\delta$, by varying the external excitation (bias $P$ ) of the cavity. Equation (6) correctly captures the variation of the RR frequency due to the velocity changes. Indeed, as shown in Fig. 1(c,e), the RR frequencies observed in the numerical spectral evolutions show a remarkable agreement with the prediction from Eq. (6), reported as vertical dashed lines in Fig. 1(c,e). Such agreement is found in a wide range of the parameters 

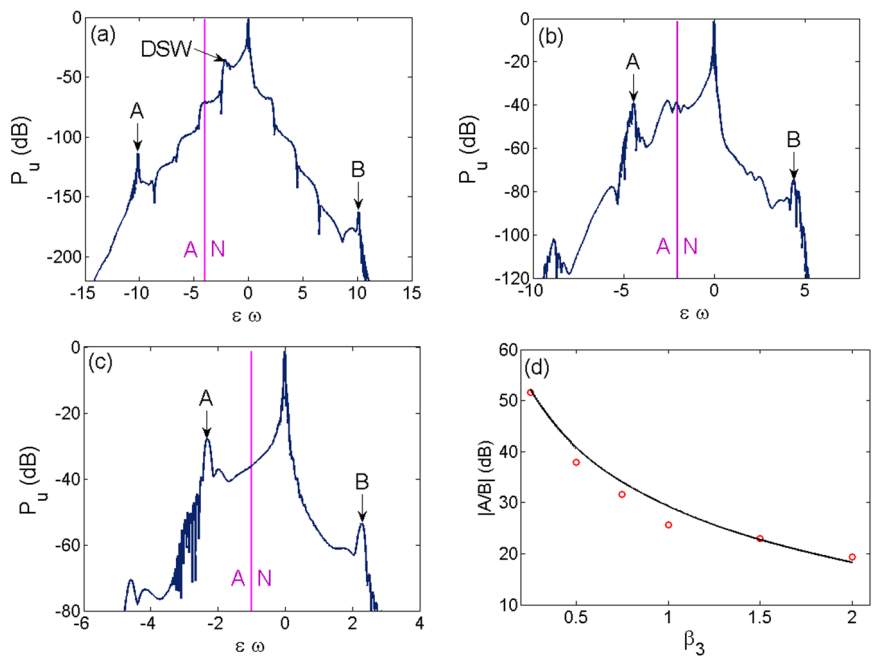

Fig. 2. (Color online) (a-c) Output spectra $(z=8)$ produced by the initial step-like excitation [as in Fig. 1(a) with $P_{u L}=0.06, P_{u H}=0.41$ ] for different values of TOD: (a) $\beta_{3}=0.25$; (b) $\beta_{3}=0.5$; (c) $\beta_{3}=1$. (d) RR sideband ratio $|A / B|$ versus $\beta_{3}$ : $\operatorname{dots}$ (numerics), solid line [Eq. (7)].

including larger values of loss $\alpha$, where the shock waves present a smoother front and less pronounced oscillatory structure, yet presenting a well-defined velocity [23].

The RR frequency always appears in the anomalous GVD, being red-shifted for $\beta_{3}>0$ (as in Fig. 1), or viceversa blue-shifted for $\beta_{3}<0$ (case not shown). For relatively low values of $\beta_{3}\left(\left|\beta_{3}\right|<0.3\right)$, the output spectrum [see Fig. 2(a)] shows a complex structure characterised by low frequency peaks associated with the DSW periodicity, and a single prominent RR peak [labeled A in Fig. 2(a)]. The conjugate (mirror-image) frequency, produced via four-wave mixing and labeled B in Fig. $2(\mathrm{a})$, remains negligible, being $50 \mathrm{~dB}$ below the A-peak. However, when $\beta_{3}$ increases, the main $\mathrm{RR}$ peak grows larger and moves at lower frequency detunings, thus enhancing the four-wave mixing, as shown in Fig. 2(b,c) for $\beta_{3}=0.5,1$. The results of this type of analysis are summarised in Fig. 2(d), where we report the ratio $|A / B|$ (in $\log$ units) against the TOD coefficient $\beta_{3}$. The numerical results (open dots) show a satisfactory agreement with the prediction from Eq. (7).

It is worth discussing the differences between the phase-matching relation Eq. (6) and those which characterise other regimes. First, comparing with the case of radiating DSW in free (cavity-less) propagation discussed in Refs. [20,21], one can notice that, here, the $\mathrm{RR}$ phase-matching is affected by the cavity detuning, and the nonlinear term $P_{u H}$ is basically constrained by the steady state value on the upper branch. Viceversa, if one compares with the RR shed by cavity solitons in the same type of structure [6,7], Eq. (6) is expected to hold valid in this case too. There is, however, a major difference in terms of quantitive impact of the velocity term. This term is indeed very important for shock waves which propagate with characteristic velocities which are essentially determined by the jump and differ dramat-
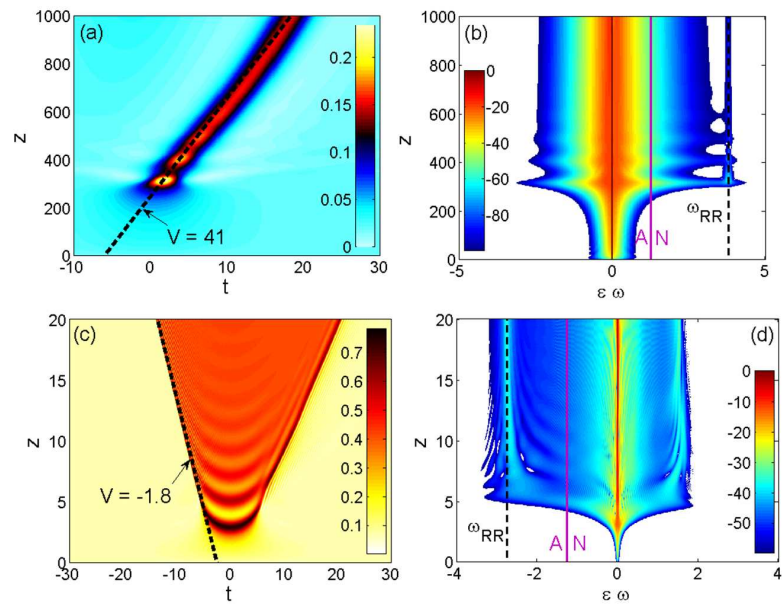

Fig. 3. (Color online) Temporal (a,c) and spectral (b,d) evolutions. The soliton regime (anomalous GVD, $\beta_{2}=$ $-1, \varepsilon=1, \delta=0.065)$ in panels (a,b), is contrasted with the shock regime (normal GVD, $\beta_{2}=1$ ), $\varepsilon=0.1, \delta=$ $\pi / 10)$ in panels $(\mathrm{c}, \mathrm{d})$. Here $\beta_{3}=0.8$ and $\alpha=0.03$. In panels $(b, d)$ vertical lines correspond to ZDW separating anomalous (A) and normal (N) GVD domains; dashed lines stand for the value of $\varepsilon \omega_{R R}$ calculated from Eq. (6).

ically from the natural group-velocity of light, so that $V$ is a small quantity. Conversely the group delay suffered by solitons is much smaller, or in other words $V$ is much larger. As such, the soliton group delay has a much weaker impact on the phase-matching of RR. This is explicitly shown in Fig. 3, where we compare the soliton evolution (pump in the anomalous GVD, $\beta_{2}=-1$ ) with the case of the shock wave (pump in the normal GVD, $\beta_{2}=1$ ) under similar operating conditions (bistable cavity with same loss and TOD coefficients $\alpha$ and $\beta_{3}$ ). In order to describe realistic excitation conditions, we integrate Eq. (1) in both cases by setting $P(t)=\left|\sqrt{P_{b}}+\sqrt{P_{p}} \operatorname{sech}(t)\right|^{2}$, where $P_{b}$ is the normalised bias power and $P_{p}$ stand for an activation power $[5,23,24]$. The latter is chosen to exceed the first knee of the bistable response so to make the highly transmissive state of the cavity easily accessible. The RR is generated in both cases [see spectra in Fig. 3(b,d)] at frequencies which are accurately described by Eq. (6), once we employ the velocities (i.e., slope of dashed lines) extrapolated from the time-domain evolutions in Fig. 3(a,c). As one can notice by comparing Fig. 3(c) for the shock wave and Fig. 3(a) for the soliton case, the former experiences a delay $d t=d z / V$ which is more than one order of magnitude larger than that of the soliton.

Finally, it is also important to emphasize that the RR expected from shock waves in a passive cavity is not necessarily the only mechanism that affects the spectral reshaping. Indeed the lower branch of the steady state is modulationally unstable [12], implying that a continuous wave can break up while propagating in the cavity. When the activation pulse lies below the first bistable knee, as shown in Fig. 4(a), the role of MI turns out to be crucial. It is indeed MI that allows for the formation of shock waves, inducing the initial break-up that, at a 

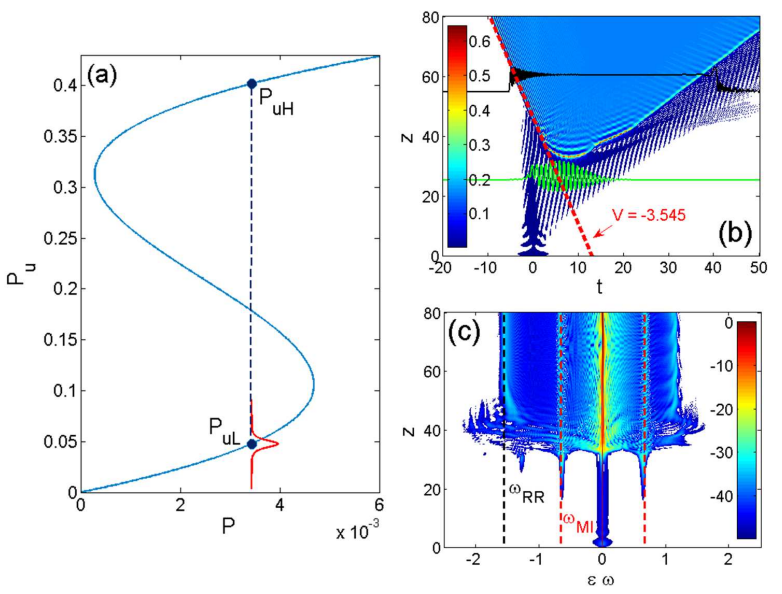

Fig. 4. (Color online). RR generated by an MI-assisted shock wave: (a) bistable response and external driving waveform (red curve) biasing the cavity over the lower branch (blue dot, $P_{u}=P_{u L}$ ); (b) temporal evolution (snapshots before and after the point of breaking $z \simeq 50$ are superimposed). Slope of red dashed line gives $V$; (c) spectral evolution with highlighted spectral peaks due to MI and RR (dashed black line from Eq. (6). Parameters: $\left.\varepsilon=0.1, \beta_{3}=2, \delta=\pi / 10, \alpha=0.03\right)$.

later stage, allows for the formation of pulses that reach the upper branch and steepen. This is clearly shown in the temporal evolution displayed in Fig. 4(b). Note that the relatively large TOD $\left(\beta_{3}=2\right)$ simulated in this case is responsible for the the strong symmetry-breaking in the time domain. TOD induces also the leading edge of such MI-assisted shock wave to radiate. In this regime the spectrum shown in Fig. 4(c) exhibits a complicated structure where multiple spectral peaks due to MI and RR coexist and can be clearly distinguished. Equation (6) gives a correct estimate for the RR frequency in this case too, provided that $P_{u H}$ corresponds to the point on the upper branch shown in Fig. 4(a), which turns out to coincide with to the upper state of the front.

We expect the RR effects from shock waves to be visible in fiber cavities. For instance $\varepsilon=0.1$ can be achieved in a cavity $L=100 \mathrm{~m}$ long $[5,24]$ with $L_{d}=L / \varepsilon^{2}=10$ $\mathrm{km}$, i.e. with $T_{0}=3.2 \mathrm{ps}$ and a GVD $k^{\prime \prime}=1 \mathrm{ps}^{2} / \mathrm{Km}$. In this case, $\beta_{3}=0.5$ corresponds to a TOD $\partial_{\omega}^{3} k=0.15$ $\mathrm{ps}^{3} / \mathrm{Km}$, and the RR frequency detuning turns out to be $f_{R R}=\omega_{R R} /\left(2 \pi T_{0}\right) \simeq 2.5 \mathrm{THz}$. The required powers scale as $1 / \gamma L$, e.g. with $\gamma=2.6(\mathrm{~W} \mathrm{~km})^{-1}, P=4 \times 10^{-3}$ yields $P_{i n}=P /(2 \alpha \gamma L) \simeq 270 \mathrm{~mW}$.

In summary, we have shown that, in passive resonators pumped close to the ZDW, shock waves feature the RR of dispersive waves. At variance with cavity solitons, the velocity of the shock front plays a decisive role, strongly affecting the radiated frequencies. RR can coexist with MI spectral peaks. These finding can also be important in light of the possibility to exploit wave breaking for comb generation $[27,28]$.

Grants from Italian Ministry of University and Research (MIUR, grant PRIN 2012BFNWZ2) and French National Research Agency (ANR, grant TOPWAVE) are gratefully acknowledged.

\section{References}

1. P. K. A. Wai, C. R. Menyuk, H. H. Chen, and Y. C. Lee, Opt. Lett. 12, 628 (1987).

2. D. V. Skryabin and A. V. Gorbach, Rev. Mod. Phys. 82, $1287(2010)$.

3. N.Y. Joly, J. Nold, W. Chang, P. Hölzer, A. Nazarkin, G. K. L. Wong, F. Biancalana, and P. St. J. Russell, Phys. Rev. Lett. 106, 203901 (2011).

4. P. Colman, S. Combrié, G. Lehoucq, A. de Rossi, and S. Trillo, Phys. Rev. Lett. 109, 093901 (2012).

5. F. Leo, S. Coen, P. Kockaert, S.-P. Gorza, P. Emplit, and M. Haelterman, Nature Photonics 4, 471 (2010).

6. C. Milian and D. V. Skryabin, Opt. Express 22, 3732 (2014).

7. J. K. Jang, S. G. Murdoch, S. Coen, and M. J. Erkintalo, "Observation of dispersive wave emission by temporal cavity solitons", CLEO 2013, paper FTh1D.2.

8. S. Wang, H. Guo, X. Bai, and X. Zeng, Opt. Lett. 39, 2880 (2014).

9. P. Parra-Rivas, D. Gomila, F. Leo, S. Coen, and L. Gelens, Opt. Lett. 39, 2971 (2014).

10. L. A. Lugiato and R. Lefever, Phys. Rev. Lett. 58, 2209 (1987).

11. M. Haelterman, S. Trillo, and S. Wabnitz, Opt. Lett. 17, 745 (1992).

12. M. Haelterman, S. Trillo, and S. Wabnitz, Opt. Commun. 91, 401 (1992).

13. A. B. Matsko, A. A. Savchenkov, W. Liang, V. S. Ilchenko, D. Seidel, and L. Maleki, Opt. Lett. 36, 2845 (2011).

14. S. Coen, H. G. Randle, T. Sylvestre, and M. Erkintalo, Opt. Lett. 38, 37 (2013).

15. Y. K. Chembo and C. R. Menyuk, Phys. Rev. A 87, 053852 (2013).

16. T. Hansson, D. Modotto, and S. Wabnitz, Phys. Rev. A 88, 023819 (2013).

17. J. E. Rothenberg and D. Grischkowsky, Phys. Rev. Lett. 62, 531 (1989).

18. D. Anderson, M. Desaix, M. Lisak, and M. L. QuiroigaTeixeiro, J. Opt. Soc. Am. B 9, 1358 (1992).

19. J. Fatome, C. Finot, G. Millot, A. Armaroli, and S. Trillo, Phys. Rev. X 4, 021022 (2014).

20. M. Conforti and S. Trillo, Opt. Lett. 38, 3815 (2013).

21. M. Conforti, F. Baronio, and S. Trillo, Phys. Rev. A 89, 013807 (2014).

22. K. E. Webb, Y. Q. Xu, M. Erkintalo, and S. G. Murdoch, Opt. Lett. 38, 151 (2013).

23. S. Malaguti, G. Bellanca, S. Trillo, Opt. Lett. 39, 2475 (2014).

24. F. Leo, A. Mussot, P. Kockaert, P. Emplit, M. Haelterman, and M. Taki, Phys. Rev. Lett. 110, 104103 (2013).

25. M. J. Schmidberger, D. Novoa, F. Biancalana, P. St. J. Russell, and N. Y. Joly, Opt. Express 22, 3046 (2014).

26. D. V. Skryabin and A. V. Yulin, Phys. Rev. E 72, 016619 (2005).

27. R. Wu, V. Torres-Company, D. E. Leaird, and A. M. Weiner, Opt. Express 21, 6045 (2013).

28. W. Liang, A. A. Savchenkov, V. S. Ilchenko, D. Eliyahu, D. Seidel, A. B. Matsko, and L. Maleki, Opt. Lett. 39, 2920 (2014). 


\section{Informational Fifth Page}

\section{References}

1. P. K. A. Wai, C. R. Menyuk, H. H. Chen, and Y. C. Lee, "Soliton at the zero-group-dispersion wavelength of a single-model fiber", Opt. Lett. 12, 628 (1987).

2. D. V. Skryabin and A. V. Gorbach, "Looking at a soliton through the prism of optical supercontinuum", Rev. Mod. Phys. 82, 1287 (2010).

3. N.Y. Joly, J. Nold, W. Chang, P. Hölzer, A. Nazarkin, G. K. L. Wong, F. Biancalana, and P. St. J. Russell, "Bright Spatially Coherent Wavelength-Tunable DeepUV Laser Source Using an Ar-Filled Photonic Crystal Fiber", Phys. Rev. Lett. 106, 203901 (2011).

4. P. Colman, S. Combrié, G. Lehoucq, A. de Rossi, and S. Trillo, "Blue Self-Frequency Shift of Slow Solitons and Radiation Locking in a Line-Defect Waveguide", Phys. Rev. Lett. 109, 093901 (2012).

5. F. Leo, S. Coen, P. Kockaert, S.-P. Gorza, P. Emplit, and M. Haelterman, "Temporal cavity solitons in onedimensional Kerr media as bits in an all-optical buffer", Nature Photonics 4, 471 (2010).

6. C. Milian and D. V. Skryabin, "Soliton families and resonant radiation in a micro-ring resonator near zero groupvelocity dispersion", Opt. Express 22, 3732 (2014).

7. J. K. Jang, S. G. Murdoch, S. Coen, and M. J. Erkintalo, "Observation of dispersive wave emission by temporal cavity solitons", CLEO 2013, paper FTh1D.2.

8. S. Wang, H. Guo, X. Bai, and X. Zeng, "Broadband Kerr frequency combs and intracavity soliton dynamics influenced by high-order cavity dispersion", Opt. Lett. 39, 2880 (2014).

9. P. Parra-Rivas, D. Gomila, F. Leo, S. Coen, and L. Gelens, "Third-order chromatic dispersion stabilizes Kerr frequency combs", Opt. Lett. 39, 2971 (2014).

10. L. A. Lugiato and R. Lefever, "Spatial dissipative structures in passive optical systems", Phys. Rev. Lett. 58, 2209 (1987).

11. M. Haelterman, S. Trillo, and S. Wabnitz, "Additivemodulation-instability ring laser in the normal dispersion regime of a fiber", Opt. Lett. 17, 745 (1992).

12. M. Haelterman, S. Trillo, and S. Wabnitz, "Dissipative modulation instability in a nonlinear dispersive ring cavity," Opt. Commun. 91, 401 (1992).

13. A. B. Matsko, A. A. Savchenkov, W. Liang, V. S. Ilchenko, D. Seidel, and L. Maleki, "Mode-locked Kerr frequency combs", Opt. Lett. 36, 2845 (2011).

14. S. Coen, H. G. Randle, T. Sylvestre, and M. Erkintalo, "Modeling of octave-spanning Kerr frequency combs using a generalized mean-field Lugiato-Lefever model", Opt. Lett. 38, 37 (2013).

15. Y. K. Chembo and C. R. Menyuk, "Spatiotemporal Lugiato-Lefever formalism for Kerr-comb generation in whispering-gallery-mode resonators", Phys. Rev. A 87, 053852 (2013).

16. T. Hansson, D. Modotto, and S. Wabnitz, "Dynamics of the modulational instability in microresonator frequency combs", Phys. Rev. A 88, 023819 (2013).

17. J. E. Rothenberg and D. Grischkowsky, "Observation of the formation of an optical intensity shock and wavebreaking in the nonlinear propagation of pulses in optical fibers", Phys. Rev. Lett. 62, 531 (1989).
18. D. Anderson, M. Desaix, M. Lisak, and M. L. QuiroigaTeixeiro, "Wave breaking in nonlinear-optical fibers", J. Opt. Soc. Am. B 9, 1358 (1992).

19. J. Fatome, C. Finot, G. Millot, A. Armaroli, and S. Trillo, "Observation of optical undular bores in multiple four-wave mixing", Phys. Rev. X 4, 021022 (2014).

20. M. Conforti and S. Trillo, "Dispersive wave emission from wave breaking", Opt. Lett. 38, 3815 (2013).

21. M. Conforti, F. Baronio, and S. Trillo, "Resonant radiation shed by dispersive shock waves", Phys. Rev. A 89, 013807 (2014).

22. K. E. Webb, Y. Q. Xu, M. Erkintalo, and S. G. Murdoch, "Generalized dispersive wave emission in nonlinear fiber optics", Opt. Lett. 38, 151 (2013).

23. S. Malaguti, G. Bellanca, S. Trillo, "Wave-breaking in coherently driven passive cavities", Opt. Lett. 39, 2475 (2014).

24. F. Leo, A. Mussot, P. Kockaert, P. Emplit, M. Haelterman, and M. Taki, "Nonlinear Symmetry Breaking Induced by Third-Order Dispersion in Optical Fiber Cavities", Phys. Rev. Lett. 110, 104103 (2013).

25. M. J. Schmidberger, D. Novoa, F. Biancalana, P. St. J. Russell, and N. Y. Joly, "Multistability and spontaneous breaking in pulse-shape symmetry in fiber ring cavities", Opt. Express 22, 3046 (2014).

26. D. V. Skryabin and A. V. Yulin, "Theory of generation of new frequencies by mixing of solitons and dispersive waves in optical fibers", Phys. Rev. E 72, 016619 (2005).

27. R. Wu, V. Torres-Company, D. E. Leaird, and A. M. Weiner, "Supercontinuum-based 10-GHz flat-topped optical frequency comb generation", Opt. Express 21, 6045 (2013).

28. W. Liang, A. A. Savchenkov, V. S. Ilchenko, D. Eliyahu, D. Seidel, A. B. Matsko, and L. Maleki, "Generation of a coherent near-infrared Kerr frequency comb in a monolithic microresonator with normal GVD", Opt. Lett. 39, 2920 (2014). 